\title{
Review of Aircraft Aerodynamic Force Parameters Identification based on the Intelligent Algorithm
}

\author{
YUFEI WANG \\ Department of Aviation Life-savingAviation University \\ of Air Force \\ Changchun 130022, China \\ aemail:19046820@qq.com \\ bemail: chuanshuo@vip.99.com
}

\author{
JIACUN XU ${ }^{1, a}$, Shen $\mathrm{Ge}^{1, \text { a }}$ and Chen $\mathrm{Lu}^{1, \mathrm{a}}$ \\ Department of Aviation Life-savingAviation University \\ of Air Force \\ Changchun 130022, China \\ e-mail: chuanshuo@vip.99.com
}

\begin{abstract}
Aerodynamic force parameter identification is the main measure of aerodynamic numerical simulation and wind tunnel test results verification. The wide use of intelligent control in the control field provides a new way to resolve the problem for system identification.Due to the global search optimization ability of intelligent algorithm, it can effectively improve precision and efficiency of aircraft aerodynamic force parameter identification. This paper gives a brief review of system identification method based on intelligent algorithm and its application in aircraft aerodynamic force parameters identification, including genetic algorithm, particle swarm optimization and artificial colony algorithm, etc. In this paper, the research work will provide useful technology reference for the aircraft aerodynamic force parameter identification.
\end{abstract}

Keywords- Aerodynamic Force Parameter Identification; Genetic Algorithm; Particle Swarm Optimization; Artificial Bee Colony Algorithm t (key words).

\section{INTRODUCTION}

Aircraft aerodynamic force parameter identification is the most mature developed field in the aircraft system identification which has been successfully used in manned spacecraft, tactical missiles and strategic missile[1-3]. Aircraft aerodynamic force parameter identification is to use the aircraft as a dynamic system, using system identification theory and technology, which establishes a mathematic model of the aircraft aerodynamic force and the aerodynamic force parameter values are obtained, with the measured data in flight test by the aircraft. With the fast development of modern control theory, mathematical statistics and electronic equipment,aircraft aerodynamic force parameter identification has gained the leap development in the application of flight test data analysis, and is playing a more and more important role in aircraft design.

This paper firstly describes the maximum likelihood identification algorithm that is widely used in engineering, and then introduces the aerodynamic parameter identification application of the genetic algorithm, particle swarm optimization and artificial colony algorithm and summarizes the general steps of the aerodynamic parameter identification for the intelligent algorithm.

\section{AERODYNAMIC PARAMETER IDENTIFICATION OF MAXIMUM LIKELIHOOD METHOD}

At present, the most widely used aerodynamic parameter identification method in engineering is maximum likelihood method, which changes the parameter identification problem into an optimization problem, through the optimization,aerodynamic model parameter values can be selected, at last making the deviation value between the model output and the measured values very small. In solving this optimization problem, the general used one is the gradient structure optimization method based on objective function, such as the steepest descent method and conjugate gradient method, etc.System identification theory proves that the maximum likelihood estimation of parameters is asymptotically unbiased, asymptotically consistent and asymptotically efficient. Thirty years of engineering practice also confirmed that the maximum likelihood method is the most efficient and practical method of aerodynamic parameter identification.

Listed above are the general steps using maximum likelihood method to make the aerodynamic parameter identification, we can see, the maximum likelihood method has the advantage that the method is simple; the amount of calculation is small.

the maximum likelihood method need to make the derivation of the identification parameters, gradient information is hard to be obtained when the objective function is discontinuous, and when dimension parameters is increased, it is easy to appear unstable pathological phenomenon; This optimization method, on the other hand, requires a high degree of the selection of initial value, the optimization results often tends to be near the local optimal solution added the initial value, in this sense, the maximum likelihood algorithm is only a local search algorithm, so it cannot not guarantee to find the optimal solution. For the current research focus of the aerodynamic parameter identification, such as high Mach number, nonlinear and unsteady aerodynamic parameter identification, the limitation of the identification method based on gradient algorithm is more prominent. 


\section{INTELLIGENT ALGORITHMS' APPLICATION IN AERODYNAMIC FORCE PARAMETER IDENTIFICATION}

At present, as a new evolutionary computation method, the intelligent algorithm is gaining more and more attention from researchers both at home and abroad, compared with a variety of adaptive random search algorithm, it achieves the search of optimal solution to optimization problem through individual interaction and competition between species. The basic idea of intelligent algorithm is to simulate behavior of process into the evolution or feeding process of the individuals, using some model of search space to simulate the individuals in nature, and at the same time, it measures the objective function to solve the problem into the individual's ability to adapt to the environment; it makes an analogy between the individual evolution or foraging process and the iterative process that the good feasible solution replaces the poor feasible solution in the search and optimization process. Therefore, intelligent algorithm is a kind of iterative search algorithm with "generate+test" characteristics.At the same time, the intelligent algorithm often includes two steps: global step and local step. Local step is the application of local optimization algorithm, while the global step is designed to ensure the solution will move to the global optimal solution area. Therefore, intelligent algorithm is also a kind of global optimization algorithm.

\section{A. Genetic algorithm}

Genetic algorithm is proposed by Professor Holland from the University of Michigan in 1975, and then the DeJong, Goldberg et al., summed it up, and finally forming a kind of simulated evolutionary algorithm. Genetic algorithm is a self-organizing and adaptive artificial intelligence technology simulating natural biological evolution process and mechanism solving extreme problems, its basic idea is to simulate natural genetic mechanism and evolution of process to search the optimal solution algorithm with a solid biological basis.

The structure of genetic algorithm:

(1)Initialization:It is to determine the population size, crossover probability, and mutation probability and terminate evolution rule, and the parameters to be identified will be showed as the genotype series data structure in the genetic space, generating initial population, and calculating the fitness value of each individual.

(2) Local optimization operation. Local optimization algorithm is used to strengthen local optimization ability of genetic algorithm. Specific operation is to randomly select a gene from each chromosome in population, after the perturbation, the adaptive value for the new individual can be got, according to the simulation local optimization algorithm, it is to determine whether gene value after perturbation can be used instead of the original value.

(3) Individual selection, copy operation: for the group after local optimization operation, selection operator is employed to regenerate the individual use, namely also the matrix.
(4) The generated matrix from the last step is made single-point crossover on the basis of crossover probability and mutation operation on the basis of mutation probability, and then the new population is generated.

(5)The individual with the largest adaptive value in the populations is found out to determine whether a calculation has convergence and if it occurs, the process will stop, otherwise, it will return to step (2).

Because the genetic algorithm has a better global optimization ability, AIAA2011-6359 ${ }^{[4]}$ apply the gradient optimization algorithm and genetic algorithm to a missile aerodynamic parameter identification and make a comparison between the two, as for some serious coupling aerodynamic parameters, genetic algorithm has a better recognition result than gradient optimization algorithm.

As a kind of adaptive global optimization probability search algorithm formed by a genetic and evolutionary process of simulated biology in the natural environment,genetic algorithm is applied to aircraft aerodynamic parameter identification field, compared with the traditional optimization algorithms of the gradient type, there are mainly the following differences:

(1) The genetic algorithm processing object is parameters code set rather than the problem parameters itself, in the aerodynamic parameter identification, its processing object is the parameters of the code set to be identified matching with the parameters, which is a single chromosome containing a set of genes;

(2) Genetic algorithm begins to search not from a single point, but from a point group, which is better to overcome the shortcomings that the traditional gradient optimization algorithm depends on the iterative initial value.

(3) Genetic algorithm employs the assessment information of the objective function, namely, the adaptive information, without the help of the target function continuity or other auxiliary information;

(4) The genetic algorithms use probability transfer rules, rather than a deterministic criterion, and this also reflects the genetic algorithm of global optimization ability.

\section{B. Particle swarm algorithm}

Particle swarm algorithm is proposed by Kennedy and Eberhart ${ }^{[5]}$ in 1995 which is analog birds foraging behaviors based on global random search strategy of bionic intelligent algorithm. The location - speed model is employed, and each particle represents a candidate in the solution space, the merits degree of the solution is determined by the fitness function, while, the fitness function is defined by the optimization guidelines ${ }^{[6]}$.Particle swarm optimization algorithm provides a generic framework for solving complex system optimization problems, independent on specific fields of the problem, at the same time it is to solve nonlinear, nondifferentiable and complex multimodal function optimization problem with strong robustness and global optimization ability.

The structure of the particle swarm algorithm: 
(1) The initialization parameter. Random position and velocity of the particle swarm are initialized.

(2) Fitness value of each particle is calculated.

(3) For each particle, its adaptive value and that of the experienced best position are compared, if it is good, then we use it as the current best position.

(4) For each particle, its adaptive value and that of the global experienced best position are compared, if it is good, then we use it as the current global best position.

(5) According to the speed and position equation, the velocity and position of the particle are compared.

(6)If it cannot meet the end condition, then we can go back to (2).

Particle swarm algorithm is a kind of bionic intelligent optimization algorithm through collaboration and information share between individuals in the group to find the optimal solution, the same with other intelligent algorithms, particle swarm algorithm also adopts the concept of "community" and "evolution" according to the size of individual adaptive value. But not like genetic algorithm, which makes evolutionary operators for individuals, particle swarm algorithm deems every individual in the search space as the particle without quality and volume, and flies in the search space at a certain speed, so the speed will be dynamically adjusted by the individual's and group's flight experience. Compared with the traditional gradient optimization algorithm, the initial position and velocity of particles for particle swarm algorithm is randomly set, which avoids the effect on the results of initial value selection in the gradient algorithm; At the same time, it uses fitness function of each particle to operate, without continuous objective function and so on; And it uses probability transfer rules to search randomly.

\section{Artificial bee colony algorithm}

Artificial bee colony algorithm is proposed by Turkish academics Karaboga ${ }^{[7]}$ which is a bionic intelligent calculation method that simulates bee colony to find good bee sources, and the algorithm has strong global convergence, which uses the following bees to lead the bee according to probability choice, and it helps algorithm resource to make the reasonable allocation, accelerate the iteration speed, at the same time, the scout bees are used to help algorithm to jump out of local optimal solution, finally the global optimal solution can be found. The algorithm increases the diversity of the solution, to some extent; it overcomes the shortcomings that are easy to premature stagnation and so on. Therefore, artificial swarm algorithm is suitable for solving complex nonlinear and multidimensional space optimization problem $^{[8]}$.

Artificial swarm algorithm structure:

(1)Initialization colony can randomly generate feasible solution and calculate the fitness of each solution vectors and regard the large adaptive value of solution as the initial employment bee populations.
(2)For the employment bees in step K, they search new flower honey in the field near the current location vector

(3)In the employed bees, the greedy selection operator is employed to search the vector quantity of the new location and in the original vector the better fitness is selected to be in a group of the next generation. Greedy selection operator can ensure the populations to retain an elite individual, which makes the evolution direction, not retreat.

(4)The following honeybees choose a bee according to the yield value of bee populations, and also search for the new flower nectar source in the field.

(5)Step(2)and(3)are repeated, when the search number around a bee mining location reaches a certain threshold and a better location still cannot be found, the position of the bees should be randomly chosen.

This purpose is to enhance the diversity of population, prevent the population from a local optimum, and this is an important aspect where artificial swarm algorithm is different from other algorithm, the step clearly makes the probability of population to search the optimal solution improved, and the effect is better.

(6) if the stop condition can be met, then the iteration will be stopped, and the optimal adaptive value and relevant optimal individual bees will be outputted, namely the value of the parameter set to be identified, or it will turn to(2).

As a kind of stochastic global optimization algorithm,inspired and proposed by bees foraging behavior, compared to genetic algorithm, particle swarm algorithm, and other intelligent optimization algorithms, artificial swarm algorithm's prominent advantage is that there are both global and local search in each iteration, and therefore to find the optimal solution with greater probability, thereby largely avoiding the local optimum.

\section{The overall plan of intelligen algorithm for aerodymamic parameter identification}

In the sections above, all kinds of intelligent algorithms the application in aerodynamic parameter identification have been described, and this section mainly illustrates the overall plan of intelligent algorithm for aerodynamic parameter identification ${ }^{[9]}$.

In the process of aircraft aerodynamic parameter identification, first of all, aircraft controllers such as rudder are sent a excitation signal according to the different object recognition.Different control input can motivate the different movement modes of vehicle dynamics system, and the test data including the information to be estimated parameters is not the same, which affects the aerodynamic parameters identification and the recognition accuracy. commonly used input signalin system identification is step, dipole square wave, "3211", frequency scan, etc. The input excitation signal can use the integral to gain a series of observations based on the aircraft dynamics model and dynamic model ${ }^{[10]}$, such as speed, angle of attack, sideslip angle and pitching angle speed, overload and other time course, and the observation time is considered to be the quantity of true value sparked by the aerodynamic parameters to be 
identified, so it is marked as $\phi_{m i}(t)$.We can take two of them according to the different recognition objects as for the specific time course of the specific amount of observation

Then the same excitation signal can be given, $\mathrm{n}$ aerodynamic parameters to be identified are taken as containing $\mathrm{n}$ genes in a chromosome or $\mathrm{n}$ particles or $\mathrm{n}$ optimal source of honey ${ }^{[11]}$, and with the help of the corresponding intelligent iterative algorithm above, and gradually approximate global optimal solution can be given, and here the global optimal solution is the true value of aerodynamic parameters to be identified. Gradually, based on the approximating true value of aerodynamic parameters to be identified, we could get a series of observations based on and dynamic model with integral, noting for $\phi_{c i}(t)$.

Finally, we make the deviation between the observation time history $\phi_{m i}(t)$ and the true value by intelligent algorithm optimization minimum, namely we make the following objective function reach the minimum amount.

$$
J=\sum \int\left[\varphi_{c i}(t)-\varphi_{m i}(t)\right]^{2} d t
$$

We take the adaptive value $F$ of each intelligent algorithm as the reciprocal form of the objective function, namely:

$$
F=\frac{1}{J+\varepsilon}(\varepsilon \text { is a number of smaller value })
$$

The true value of the aerodynamic parameter to be identified comes to the global optimal point corresponding to objective function, namely, $J=0$,so the biggest fitness value should eventually converge to $\frac{1}{\varepsilon}$.

Particular, it is essentially a kind of intelligent algorithm with random heuristic search algorithm. So according to the expert experience, the limitation for possible search scope of aerodynamic parameters identification is very important, especially, the smaller sensitivity parameters to be identified, because it is more sensitive to the search space, search range should be set according to expert experience.

\section{CONCLUSION}

Intelligent algorithm has a broad application prospect in the field of aircraft aerodynamic parameter identification because of its global search ability, low requirements for iterative initial value and without characteristics such as objective function.Part of the intelligent algorithms has been used by domestic and foreign scholars in the field of aircraft aerodynamic parameter identification, and good simulation results have been achieved. But we can also clearly see that, for the new intelligent algorithm appearred in recent years, because its occurring period is shorter, theoretical analysis is not complete, its application in aerodynamic parameter identification is in a show state, many new emerging intelligent algorithm is little or even not applied to the identification aircraft aerodynamic parameters, which shows that the new intelligent algorithm has broad application prospects from another aspect in the field of aircraft aerodynamic parameter identification. According to the current application status, each intelligent algorithm applied to the aircraft aerodynamic parameter identification has widespread shortcomings of the larger amount of calculation and the lower efficient calculation compared with traditional gradient algorithm.Aimed at this shortage, the focus of the next step is to combine the intelligent algorithm with the traditional gradient algorithm and various intelligent algorithms complement with each other, further speed up the local optimization ability, and improve the overall computational efficiency; At the same time, because the fitness evaluation of the intelligent algorithm and random search and other features have the obvious feature of parallelism, the present application does not make good use of the advantage of intelligent algorithm paralle computing, therefore, the design of parallel execution strategy can greatly improve the operation efficiency.

\section{REFERENCES}

[1] Wang K C, Iliff K W. Retrospective and recent examples of aircraft parameter identification at NASA Dryden Flight Research Center[J]. Journal of Aircraft 2004; 41(4): 752-764.

[2] Morelli E A, Klein V. Application of system identification to aircraft at NASA Langley Research Center[J]. Journal of Aircraft 2005; 42(1): 12-25.

[3] Jategaonkar R, Fischenberg D, von Gruenhagen W. Aerodynamic modeling and system identification from flight data-recent applications at DLR[J]. Journal of Aircraft 2004; 41(4): 681-691.

[4] A. Vitale, F. Corraro, M. Bernard, G. De Matteis. Unscented Kalman Filtering for Reentry Vehicle Identification in the Transonic Regime[J]. JOURNAL OF AIRCRAFT. 2009, 46(5):1649-1659.

[5] Fabio A. de Almeida. Aerodynamic Model Identification of a Fighter Trainer using Partial Orthogonal Least Squares[C]. 50th AIAA Aerospace Sciences Meeting including the New Horizons Forum and Aerospace Exposition. AIAA 2012-1049.

[6] Christopher Jouannet, Petter Krus. Modelling of High Angle of Attack Aerodynamic[C]. 25th AIAA Applied Aerodynamics Conference, AIAA 2007-4295.

[7] Adel Younis1, Jichao Gu, Zuomin Dong, Guangyao Li. Trends, Features, and Tests of Common and Recently Introduced Global Optimization Methods[J]. 12th AIAA/ISSMO Multidisciplinary Analysis and Optimization Conference. AIAA 2008-5853.

[8] Bradley T. Burchett. Aerodynamic Parameter Identification for Symmetric Projectiles: Comparing Gradient Based and Evolutionary Algorithms[J]. AIAA Atmospheric Flight Mechanics Conference. AIAA 2011-6359.

[9] WANG Xiao Peng. Aerodynamic parameter identification of flight vehicles based on adaptive genetic algorithm [J]. Journal of Astronautics, 2003, 24(3):303-308

[10] Kennedy J, Eberhart R. Particle swarm optimization[C]. Proc IEEE Int Conf on Neural Networks .Perth, 1995. 1942-1948.

[11] Mauro Pontani, Bruce A. Conway. Particle Swarm Optimization Applied to Space Trajectories[J]. Journal of Guidance, Control, and Dynamics, 2010, 33(5):1429-1441. 
